\title{
HER2 status and breast cancer therapy: recent advances Debu Tripathy
}

Address: University of Texas Southwestern Medical Center in Dallas, Physicians' Education Resource, 3500 Maple Ave, Suite 700, Dallas, TX 75219, USA

Email: debu.tripathy@utsouthwestern.edu

FI000 Medicine Reports 2009, I:20 (doi: 10.34I0/MI-20)

The electronic version of this article is the complete one and can be found at: http://FI000.com/Reports/Medicine/content/I/20

\begin{abstract}
The phenotype imparted by expression of the HER2 gene in breast cancer and progress made in modifying the disease's natural history through pharmacologically modulating its function has served as a paradigm for rationally targeted therapy and personalized medicine. About $20-25 \%$ of breast cancer cases are associated with HER2 gene amplification and overexpression, creating a distinct subtype of breast cancer that is associated with more aggressive behaviour, higher likelihood of overall and brain metastases, and differential responsiveness to certain hormonal and chemotherapeutic agents. Anti-HER2 monoclonal antibodies have led to significant improvements in survival for both advanced and early stage HER2+ breast cancer, while newer agents, including other antibodies and HER2 receptor tyrosine kinase inhibitors and signal transduction modulators, are also demonstrating clinical activity and represent further opportunities to improve curability and quality of life.
\end{abstract}

\section{Introduction and context}

Upon the recognition of the importance of HER2 amplification and overexpression in the outcome of patients with early stage breast cancer, laboratory experiments using anti-HER2 antibodies consistently showed growth inhibitory effects, presumably due to modulation of downstream signal transduction, but also potentially due to activation of the immune system against HER2 epitopes [1,2]. Phase I and II trials showed that a humanized HER2 antibody, trastuzumab (trade name Herceptin $\left.{ }^{\circledR}\right)$, given as a single agent could lead to transient - but in some cases, long-lived - responses in $15 \%$ of advanced breast cancer patients in the second line setting and $26 \%$ of patients as first line therapy [35]. A pivotal randomized trial showed that the addition of trastuzumab to standard first line chemotherapy in HER2+ advanced breast cancer can improve response rates and time to disease progression (TTP) and also improved median survival by a relative amount of $24 \%$, or an absolute median gain of 5 months, leading to the approval of this drug in HER2+ metastatic breast cancer (Figure 1) [6].
Systemic therapy used in the early stage adjuvant setting has the potential to improve long-term curability, so the logical extension of these findings was to compare the addition of trastuzumab to standard chemotherapy in patients with HER2+ early stage breast cancer, using 1 year of trastuzumab therapy - a length of time that was arbitrary, but at least supported by safety data. Careful cardiac screening and monitoring was incorporated into all four major pivotal trials done worldwide. The two North American trials [N9831 and National Surgical Bowel and Breast Program (NSABP) B-31] used a standard regimen of doxorubicin and cyclophosphamide (AC) for four cycles every 3 weeks followed by paclitaxel either every 3 weeks for four cycles or at a lower dose every week for 12 weeks with or without trastuzumab. Trastuzumab was given with paclitaxel at standard doses. The N9831 trial contained a third arm in which trastuzumab was given following paclitaxel. The Breast Cancer International Research Group (BCIRG) 006 trial used a control arm of AC followed by four cycles of docetaxel. The second arm was administered trastuzumab with docetaxel. A third arm used docetaxel and 
Figure I. HER2 detection in tumour tissue and impact of trastuzumab on survival when added to chemotherapy for advanced HER2+ breast cancer

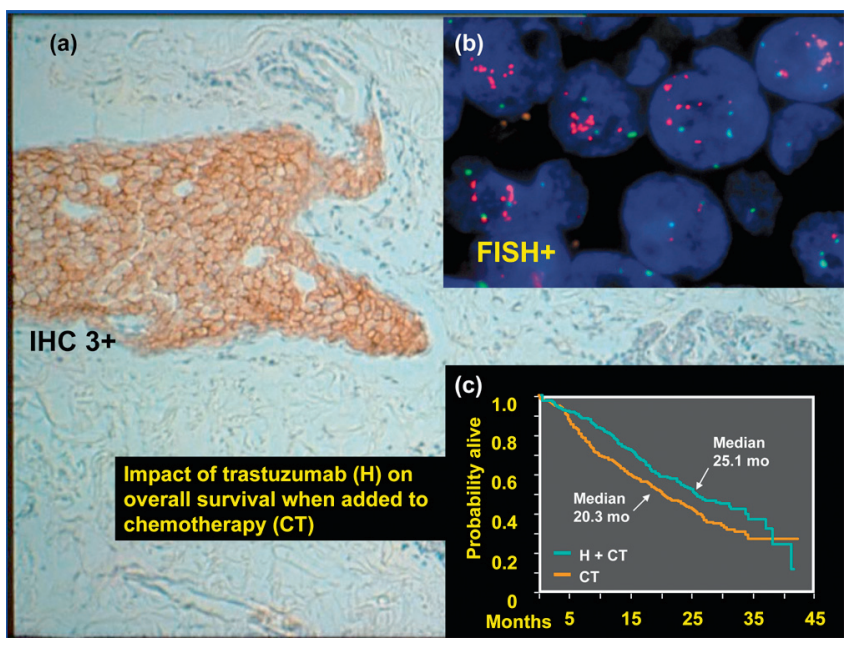

HER2 status is assessed either by (a) immunohistochemistry (IHC) level 3+, or by (b) gene amplification, defined by fluorescent in situ hybridization (FISH), where the average ratio of HER2 gene (red fluorochrome) to the centromeric portion of chromosome 17 (blue fluorochrome) is $\geq 2.2$. (c) Survival is improved with the addition of trastuzumab to chemotherapy [6].

carboplatin every 3 weeks for six cycles with trastuzumab concurrently; this arm was based on preclinical synergy noted in preclinical models with both these chemotherapeutic agents and trastuzumab and was also testing whether less cardiotoxicity might be observed with a non-anthracycline arm. The HERA 3-arm study was more permissive and allowed any pre- or postoperative chemotherapy regimen of more than four cycles compared to chemotherapy with either 1 or 2 years of trastuzumab.

All these trials were stopped early or reported at early interim analysis as the effect size was greater than expected, with hazard ratios for recurrence-free survival ranging from 0.48 to 0.67 , and absolute differences in disease-free survival at 3-4 years ranging from 5 to $12.8 \%$ [7-10]. Significant differences in overall survival were also seen in all trials, and other findings of these pivotal trials are shown on Table 1 . Symptomatic cardiac toxicity was seen in $0.4-3.8 \%$ of patients, with higher rates seen with concurrent chemotherapy that included an anthracycline, and recovery was seen in most cases [811]. Higher age, lower pre-trastuzumab cardiac ejection fraction and history of hypertension or use of antihypertensive medications were noted as risk factors for cardiac events, and most, but not all, patients recovered from these side effects $[11,12]$. Based on these data, trastuzumab has been approved with the regimens used in the North American and BCIRG 006 trials for nodepositive and high risk node-negative HER2+ breast cancer.

\section{Recent advances}

Currently, the use of trastuzumab is considered standard therapy in the first line setting for advanced HER2+ breast cancer, typically in combination with chemotherapy, but is also used as a single agent as initial or second line therapy. There is some controversy about therapy after progression on trastuzumab - the combination of lapatinib and capecitabine is approved on the basis of a randomized trial showing that this combination led to a significant prolongation of TTP [13]. However, trastuzumab plus capecitabine has also been found to prolong TTP compared to capecitabine alone in patients who had progression on trastuzumab-based therapy [14]. In the adjuvant setting, individualized decision-making that balances benefits against risks, particularly cardiomyopathy, is warranted and generally this is indicated in node-positive or high risk node-negative disease, although other tumour and patient factors (tumour grade, age, co-morbidities) all play a role. Only combinations with chemotherapy have been tested in early stage disease, so the optimal chemotherapy partner remains to be defined - in the US, anthracycline and taxane regimens are more commonly used. The use of

Table I. Pivotal HER2-targeting adjuvant trials using trastuzumab

\begin{tabular}{|c|c|c|c|c|c|c|c|c|}
\hline $\begin{array}{l}\text { Trial/experimental } \\
\text { regimen }\end{array}$ & $\mathbf{n}$ & $\begin{array}{l}\text { Median f/u } \\
\text { (years) }\end{array}$ & HR DFS & HR OS & $\begin{array}{c}\text { Absolute \% } \\
\text { difference DFS }\end{array}$ & $\begin{array}{c}\text { Absolute \% } \\
\text { difference OS }\end{array}$ & CHF (\%) & Reference \\
\hline $\begin{array}{l}\text { NSABP B-3 I/N983I: } \\
\text { AC } \rightarrow \text { TH }\end{array}$ & 3,968 & 2.9 & 0.48 & 0.65 & 12.8 at 4 years & 3.2 at 4 years & $2.5-3.8$ & {$[6,7,10]$} \\
\hline HERA: multiple $\rightarrow \mathrm{H}$ & 3,401 & 2 & 0.64 & 0.66 & 6.3 at 3 years & 2.7 at 3 years & 0.6 & [8] \\
\hline $\mathrm{BCIRG}$ 006: $\mathrm{AC} \rightarrow \mathrm{DH}$ & 3,222 & 2 & 0.61 & 0.58 & 6 at 3 years & 4 at 3 years & 1.9 & [9] \\
\hline BCIRG 006: DCaH & 3,222 & 2 & 0.67 & 0.66 & 5 at 3 years & 2 at 3 years & 0.4 & [9] \\
\hline
\end{tabular}

A, doxorubicin; Absolute \% difference DFS, absolute percentage difference in disease-free survival; Absolute \% difference OS, absolute percentage difference in overall survival; BCIRG, Breast Cancer International Research Group; C, cyclophosphamide; Ca, carboplatin; CHF, congestive heart failure (symptomatic); D, docetaxel; H, trastuzumab (Herceptin); HERA, Herceptin Adjuvant; HR DFS, hazard ratio disease-free survival; HR OS, hazard ratio overall survival; Median f/u, median follow-up; n, number of patients; NSABP, National Surgical Adjuvant Breast and Bowel Project; T, paclitaxel. 
trastuzumab alone or in combination with hormonal therapy alone has not been tested in randomized trials. Durations of less than 1 year have not been fully tested, although an underpowered Finnish study, known as the FinHer trial, showed efficacy when trastuzumab was used in combination with chemotherapy for a short period [15]. Therapy should be temporarily suspended or permanently discontinued for any clinical cardiac complications, and in some cases for subclinical decrease in ejection fraction.

Newer HER2-targeted agents such as small molecule kinase inhibitors are being tested, with lapatinib now being tested both in advanced and early stage disease, and HKI-272 in early phase trials for HER2+ advanced breast cancer [16]. Pertuzumab, a new antibody that inhibits dimerisation of HER2 with other HER family members, has also shown activity in trastuzumabrefractory disease [17], as has trastuzumab-DM1, an immunotoxin $[18,19]$. Combining trastuzumab with HSP90 inhibitors, which affect the trafficking and proper folding of several proteins involved in signal transduction, has also yielded responses in trastuzumab-refractory cases [20].

\section{Implications for clinical practice}

The HER2 oncogene represents an important mediator of breast carcinogensis and is a clinically relevant biomarker. Targeting HER2 with the antibody trastuzumab has been shown to improve outcome in both advanced and early stage disease and represents a standard of care that requires HER analysis of all breast cancer cases. Guidelines for HER2 interpretation and the need to use highvolume and accredited laboratories are critical [21]. Estimations of the risk of recurrence along with hazard reductions from clinical trials need to be juxtaposed against cardiac and other side effects for optimal individualized decision-making. In the advanced setting, newer HER2-targeting agents will be tested more systematically as salvage therapy, and then in first line use with the hopes that more activity, or less toxicity, might be seen. It is not clear if it is best to use a combination of HER2-directed therapies at the outset, or to wait until there is evidence of clinical resistance. Well designed tissue correlative studies may provide newer biomarkers to predict responses to specific HER2-targeted drugs and aid in decision-making as well as in identifying new targets that could be addressed pharmacologically to reverse resistance to trastuzumab or improve outcomes when added to trastuzumab.

\section{Abbreviations}

AC, doxorubicin and cyclophosphamide; BCIRG, Breast Cancer International Research Group; NSABP, National Surgical Bowel and Breast Program; TTP, time to disease progression.

\section{Competing interests}

The author declares that he has no competing interests.

\section{References}

I. Slamon DJ, Clark GM, Wong SG, Levin WJ, Ullrich A, McGuire WL: Human breast cancer: correlation of relapse and survival with amplification of the HER-2/neu oncogene. Science 1987, 235: 177-82.

2. Hudziak RM, Lewis GD, Winget M, Fendly BM, Shepard HM, Ullrich A: pl85HER2 monoclonal antibody has antiproliferative effects in vitro and sensitizes human breast tumor cells to tumor necrosis factor. Mol Cell Biol I989, 9: I I65-72.

3. Cobleigh MA, Vogel CL, Tripathy D, Robert NJ, Scholl S, Fehrenbacher L, Wolter JM, Paton V, Shak S, Lieberman G, Slamon $D$ : Multinational study of efficacy and safety of humanized anti-HER2 monoclonal antibody in women who have HER2overexpressing metastatic breast cancer that has progressed after chemotherapy for metastatic disease. J Clin Oncol 1999, I 7:2639-2648.

4. Vogel CL, Cobleigh MA, Tripathy D, Gutheil JC, Harris LN, Fehrenbacher L, Slamon DJ, Murphy M, Novotny WF, Burchmore M, Shak S, Stewart SJ, Press M: Efficacy and safety of trastuzumab as a single agent in first-line treatment of HER2-overexpressing metastatic breast cancer. J Clin Oncol 2002, 20:719-26.

5. Baselga J, Carbonell X, Castañeda-Soto NJ, Clemens M, Green M, Harvey V, Morales S, Barton C, Ghahramani P: Phase II study of efficacy, safety, and pharmacokinetics of trastuzumab monotherapy administered on a 3-weekly schedule. J Clin Oncol 2005, 23:2162-7I.

6. Slamon DJ, Leyland-Jones B, Shak S, Fuchs H, Paton V, Bajamonde A Fleming T, Eiermann W, Wolter J, Pegram M, Baselga J, Norton L: Use of chemotherapy plus a monoclonal antibody against HER2 for metastatic breast cancer that overexpresses HER2. $N$ Engl J Med 2001, 344:783-92.

7. Romond EH, Perez EA, Bryant J, Suman VJ, Geyer CE Jr, Davidson NE, Tan-Chiu E, Martino S, Paik S, Kaufman PA, Swain SM, Pisansky TM, Fehrenbacher L, Kutteh LA, Vogel VG, Visscher DW, Yothers G, Jenkins RB, Brown AM, Dakhil SR, Mamounas EP, Lingle WL, Klein $\mathrm{PM}$, Ingle JN, Wolmark N: Trastuzumab plus adjuvant chemotherapy for operable HER2-positive breast cancer. $N$ Engl J Med 2005, 353:1673-84

FI000 Factor 9.0 Exceptional

Evaluated by Ruth Keri 16 Dec 2005

8. Perez EA, Romond EH, Suman VJ, Jeong J, Davidson NE, Geyer CE, Martino S, Mamounas EP, Kauffman PA, Wolmark N; NCCTG/NSABP. Updated results of the combined analysis of NCCTG N983 I and NSABP B-3I adjuvant chemotherapy with/without trastuzumab in patients with HER2-positive breast cancer, J Clin Oncol 2007, ASCO Annual Meeting Proceedings Part I, 25 (June 20 Suppl): 6s. Abstract 512.

9. Smith I, Procter M, Gelber RD, Guillaume S, Feyereislova A, Dowsett M, Goldhirsch A, Untch M, Mariani G, Baselga J, Kaufmann M, Cameron D, Bell R, Bergh J, Coleman R, Wardley A, Harbeck N, Lopez RI, Mallmann P, Gelmon K, Wilcken N, Wist E, Sánchez Rovira P, Piccart-Gebhart MJ; HERA study team: 2-year follow-up of trastuzumab after adjuvant chemotherapy in HER2-positive breast cancer: a randomised controlled trial. Lancet 2007, 369:29-36

10. Slamon D, Eiermann W, Robert N, Pienkowski T, Martin M, Pawlicki M, Chan A, Smylie M, Liu M, Falkson C, Pinter T, Fornander T, Shiftan T, Valero V, Mackey J, Tabah-Fisch I, Buyse M, Lindsay M, Riva A, Bee 
V, Pegram M, Press M, Crown J; BCIRG 006: 2nd interim analysis phase III randomized trial comparing doxorubicin and cyclophosphamide followed by docetaxel (ACT) with doxorubicin and cyclophosphamide followed by docetaxel and trastuzumab (ACTH) with docetaxel, carboplatin and trastuzumab (TCH) in Her2neu positive early breast cancer patients. Breast Cancer Res Treat 2006, I00 (Suppl I): Abstract 52.

II. Rastogi P, Jeong J, Geyer CE, Costantino JP, Romond EH, Ewer MS, Keefe DL, Levine T, Swain SM, Wolmark N: Five year update of cardiac dysfunction NSABP B-3I, a randomized trial of sequential doxorubicin/cyclophosphamide (AC) $\rightarrow$ paclitaxel (T) vs. AC $\rightarrow$ T with trastuzumab(H). J Clin Oncol 2007, ASCO Annual Meeting Proceedings Part I, 25 (June 20 Suppl):6s. Abstract LBA5I3.

12. Perez EA, Suman VJ, Davidson NE, Sledge GW, Kaufman PA, Hudis CA, Martino S, Gralow JR, Dakhil SR, Ingle JN, Winer EP, Gelmon KA, Gersh BJ, Jaffe AS, Rodeheffer RJ: Cardiac safety analysis of doxorubicin and cyclophosphamide followed by paclitaxel with or without trastuzumab in the North Central Cancer Treatment Group N983 I adjuvant breast cancer trial. J Clin Oncol 2008, 26: |23|-38.

13. Geyer CE, Forster J, Lindquist D, Chan S, Romieu CG, Pienkowski T, Jagiello-Gruszfeld A, Crown J, Chan A, Kaufman B, Skarlos D, Campone M, Davidson N, Berger M, Oliva C, Rubin SD, Stein S, Cameron D: Lapatinib plus capecitabine for HER2-positive advanced breast cancer. N Engl J Med 2006, 355:2733-43.

FI000 Factor 6.6 Must Read

Evaluated by Debu Tripathy 7 Feb 2007, Daniel Vorobiof 6 Mar 2007, Nicola Normanno 6 Mar 2007

14. Von Minckwitz G, Zielinski C, Maarteense E, Vogel P, Schmidt M, Eidtmann H, Cufer T, de Jongh FE, Kaufmann M, Loibl: Capecitabine vs. capecitabine + trastuzumab in patients with HER2-positive metastatic breast cancer progressing during trastuzumab treatment: The TBP phase III study (GBG 26/BIG 3-05). J Clin Oncol 2008, ASCO Annual Meeting Proceedings Part I, 26:47s. Abstract 1025.

15. Joensuu $H$, Kellokumpu-Lehtinen PL, Bono P, Alanko T, Kataja V, Asola R, Utriainen T, Kokko R, Hemminki A, Tarkkanen M, Turpeenniemi-Hujanen T, Jyrkkiö S, Flander M, Helle L, Ingalsuo S, Johansson K, Jääskeläinen AS, Pajunen M, Rauhala M, Kaleva-Kerola J, Salminen T, Leinonen M, Elomaa I, Isola J; FinHer Study Investigators:
Adjuvant docetaxel or vinorelbine with or without trastuzumab for breast cancer. N Eng J Med 2006, 354:809-20.

16. Burstein HJ, Awada A, Badwe R, Dirix L, Tan A, Jacod S, Lustgarten S, Vermette J, Zacharchuk C: HKI-272, an irreversible pan erbB receptor tyrosine kinase inhibitor: preliminary phase 2 results in patients with advanced breast cancer. Breast Cancer Res Treat 2007, 106 (SuppI I):S268. Abstract 606I.

17. Gelmon KA, Fumoleau P, Verma S, Wardley AM, Conte PF, Miles D, Gianni L, McNally VA, Ross G, Baselga J: Results of a phase II trial of trastuzumab $(H)$ and pertuzumab $(P)$ in patients (pts) with HER2-positive metastatic breast cancer (MBC) who had progressed during trastuzumab therapy. J Clin Oncol 2008, ASCO Annual Meeting Proceedings Part I, 26 (May 20 Suppl):48s. Abstract 1026.

18. Beeram M, Burris HA, Modi S, Birkner M, Girish S, Tibbitts J, Holden SN, Lutzker SG, Krop IE: A phase I study of trastuzumab-DMI (T-DMI), a first-in-class HER2 antibody-drug conjugate (ADC), in patients (pts) with advanced HER2+ breast cancer (BC). J Clin Oncol 2008, ASCO Annual Meeting Proceedings Part I, 26 (May 20 Suppl):48s. Abstract 1028.

19. Holden SN, Beeram M, Krop IE, Burris HA 3rd, Birkner M, Girish S, Tibbitts J, Lutzker SG, Modi S: A phase I study of weekly dosing of trastuzumab-DMI (T-DMI) in patients (pts) with advanced HER2+ breast cancer (BC). J Clin Oncol 2008, ASCO Annual Meeting Proceedings Part I, 26(May 20 Suppl):47s. Abstract 1029.

20. Modi S, Sugarman S, Stopeck A, Linden H, Ma W, Kersey K, Johnson RG, Rosen N, Hannah AL, Hudis CA: Phase II trial of the Hsp90 inhibitor tanespimycin (Tan) + trastuzumab $(T)$ in patients (pts) with HER2-positive metastatic breast cancer (MBC). J Clin Oncol 2008, ASCO Annual Meeting Proceedings Part I, 26(May 20 Suppl):47s. Abstract 1027.

2I. Wolff AC, Hammond ME, Schwartz JN, Hagerty KL, Allred DC, Cote RJ, Dowsett M, Fitzgibbons PL, Hanna WM, Langer A, McShane LM, Paik S, Pegram MD, Perez EA, Press MF, Rhodes A, Sturgeon C, Taube SE, Tubbs R, Vance GH, van de Vijver M, Wheeler TM, Hayes DF; American Society of Clinical Oncology; College of American Pathologists: American Society of Clinical Oncology/ College of American Pathologists guideline recommendations for human epidermal growth factor receptor 2 testing in breast cancer. J Clin Oncol 2007, 25: I I8-45. 